\title{
LES « ENRÔLÉS DE FORCE » DANS LA WEHRMACHT - UN SYMBOLE DU PASSÉ MOUVEMENTÉ DES BELGES GERMANOPHONES AU XXE SIÈCLE
}

Christoph Brïll

Presses Universitaires de France | « Guerres mondiales et conflits contemporains »

2011/1 n $241 \mid$ pages 63 à 74

ISSN 0984-2292

ISBN 9782130587170

Article disponible en ligne à l'adresse :

http://www.cairn.info/revue-guerres-mondiales-et-conflits-contemporains-2011-1-page-63.htm

\section{!Pour citer cet article :}

Christoph Brüll, «Les « enrôlés de force » dans la Wehrmacht - un symbole du passé mouvementé des belges germanophones au xxe siècle », Guerres mondiales et conflits contemporains 2011/1 ( $\mathrm{n}^{\circ} 241$ ), p. 63-74.

DOI $10.3917 /$ gmcc. 241.0063

Distribution électronique Cairn.info pour Presses Universitaires de France.

(C) Presses Universitaires de France. Tous droits réservés pour tous pays.

La reproduction ou représentation de cet article, notamment par photocopie, n'est autorisée que dans les limites des conditions générales d'utilisation du site ou, le cas échéant, des conditions générales de la licence souscrite par votre établissement. Toute autre reproduction ou représentation, en tout ou partie, sous quelque forme et de quelque manière que ce soit, est interdite sauf accord préalable et écrit de l'éditeur, en dehors des cas prévus par la législation en vigueur en France. Il est précisé que son stockage dans une base de données est également interdit. 


\section{LES «ENRÔLÉS DE FORCE » DANS LA WEHRMACHT - UN SYMBOLE DU PASSÉ MOUVEMENTÉ DES BELGES GERMANOPHONES AU XX ${ }^{\mathrm{e}}$ SIĖCLE ${ }^{1}$}

\section{INTRODUCTION}

Au $\mathrm{xx}^{\mathrm{e}}$ siècle, l'histoire des 75000 Belges germanophones est celle d'un passé mouvementé ${ }^{2}$. Devenue belge à la suite du traité de Versailles, la population, prussienne depuis 1815, des deux Kreise d'Eupen (germanophone) et de Malmedy (en partie wallonne ou francophone) a vécu des conflits de loyauté résultant du nationalisme exacerbé si typique de la première moitié du siècle dernier. Le symbole de ce passé, mais aussi de ces conflits d'allégeance, ce sont les 8700 jeunes hommes recrutés par la Wehrmacht à partir du mois de novembre 1941, soit deux mois après l'octroi de la nationalité allemande à la population d'un territoire annexé de facto au Reich par un Führererlass du 18 mai 1940. Entre 3200 et 3400 d'entre eux, soit $39 \%$, ne reviendront pas, mourant au "champ d'honneur » ou dans des camps de prisonniers soviétiques ${ }^{3}$.

La terminologie a fait de ces soldats des Zwangssoldaten - des "enrôlés de force ». Ce terme est d'abord créé par les autorités gouvernementales bruxelloises pour les distinguer de leurs compatriotes flamands et wallons qui s'étaient portés volontaires dans l'armée allemande. Il est ensuite repris

1. Christoph Brüll (1979) est docteur en histoire contemporaine de la Friedrich-SchillerUniversität d'Iéna et titulaire d'un DEA en relations internationales et intégration européenne de l'université de Liège. Il est chargé de recherches du Fonds de la recherche scientifique-FNRs auprès de l'université de Liège. Ses publications portent sur l'histoire des relations belgo-allemandes, de la mémoire et de l'historiographie et sur l'histoire de la Communauté germanophone de Belgique. Il a publié Die deutschsprachigen Einheiten in der belgischen Armee zwischen den beiden Weltkriegen, Saint Vith, 2004 et Belgien und Nachkriegsdeutschland: Besatzung, Annäherung, Ausgleich 1944-1958, Essen, 2009.

2. Pour des aperçus en langue française voir Alfred Minke, "La Communauté germanophone : l'évolution d'une terre d'entre-deux ", in www.wallonie-en-ligne.net, 1995 [14.06.2010] ; Christoph Brül, «Un passé mouvementé. L'histoire de la Communauté germanophone de Belgique ", in Katrin Stangherlin (éd.), La Communauté germanophone de Belgique - Die Deutschsprachige Gemeinschaft Belgiens, Bruxelles, Éd. La Charte, 2005, p. 17-47. Une exception est formée par l'actuelle commune de La Calamine qui formait entre 1815 et 1919 le territoire de Moresnet-Neutre et qui n'a donc jamais fait partie de la Prusse.

3. Quelques données chiffrées et informations de base peuvent être trouvées chez Heinrich Toussaint, "Zwangssoldaten ", in Alfred Minke e.a. (éds.) Grenzland seit Menschengedenken, Biblio-Cassette 2 : Abhängigkeit, Eupen, 1990, $\mathrm{n}^{\circ} 74$. 
par les intéressés et les représentants de leurs associations. À travers les décennies, ce sont une connotation de victimes et le potentiel de victimisation de cette terminologie qui ont forgé l'image de ces soldats, mais aussi, à travers eux, de toute la population germanophone. Ce terme ne prend cependant pas en compte les conflits entre probelges et proallemands qui ont régi la vie politique et sociale de tout l'entre-deux-guerres. Il ne permet pas non plus de poser la question du comportement de ces soldats sur le front. En ce sens, il s'intègre dans une conception de l'histoire qui est née à la fin de la Seconde Guerre mondiale au moment de l' "épuration civique », et qui, en insistant sur le caractère "belge » du territoire et de sa population, a empêché toute approche nuancée de l'histoire, voire déformé grossièrement le passé le plus récent ${ }^{4}$. À cet égard, le rapport à la figure du soldat reflète le rapport au passé marqué par les deux guerres mondiales.

L'historiographie s'est également plutôt tardivement penchée sur ces soldats. Si une thèse suisse décrit en 1975 pour la première fois les implications juridiques et administratives de l'annexion du territoire d'EupenMalmedy par l'Allemagne en mai 1940 et donc l'enrôlement à partir des mois d'automne $1941^{5}$, il a fallu attendre 2008 pour qu'un jeune historien régional analyse pour la première fois en profondeur les mécanismes de recrutement de la Wehrmacht ${ }^{6}$. Dans l'historiographie belge, qu'elle soit flamande ou wallonne, ces soldats n'ont jusqu'à présent jamais eu leur place. Dans les statistiques de pertes belges de la Seconde Guerre mondiale les plus récentes, les 3200 jeunes hommes de la région tués ou disparus n'apparaissent pas - au contraire des volontaires flamands et wallons dans la Wehrmacht ou la ss $^{7}$. Pendant la décennie 1980, ce sont les souvenirs des intéressés qui ont fait l'objet de publications qui les présentent la plupart du temps comme des victimes innocentes du grand jeu de la politique internationale, en général, et du rattachement à l'Allemagne en 1940, en particulier ${ }^{8}$.

\section{BREF REGARD SUR L'ENTRE-DEUX-GUERRES}

Le changement de nationalité de 1920 soulève le difficile problème de faire le deuil des hommes morts pour l'Allemagne - l'ennemie de la

4. Freddy Cremer, ",Verschlusssache Geschichte“. Über den Umgang mit der eigenen Vergangenheit ", in: Cremer Freddy, Fickers Andreas, Lejeune Carlo (eds.), Spuren in die Zukunft. Anmerkungen zu einem bewegten Jahrhundert, Büllingen Lexis-Verlag, 2001, p. 9-26 (p. 23-24).

5. Martin R. Schärer, Deutsche Annexionspolitik im Westen. Die Wiedereingliederung Eupen-Malmedys

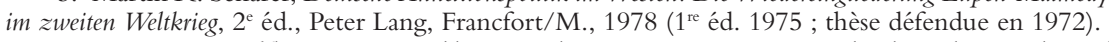

6. Peter M. Quadflieg, „Zwangssoldaten“ und „Ons Jongen“. Eupen-Malmedy und Luxemburg als Rekrutierungsgebiet der deutschen Wehrmacht im Zweiten Weltkrieg, Aix-la-Chapelle, Shaker Verlag, 2008.

7. Rudi Van Doorslaer, "L'héritage politique de la guerre et en particulier de la collaboration en Belgique, 1945-2000", in : Archives nationales Luxembourg (éds), Collaboration : Nazification ? Le cas du Luxembourg à la lumière des situations française, belge et néerlandaise. Actes du colloque international de Neumünster, mai 2006, Éd. Archives nationales, Luxembourg, 2008, p. 444-460 (p. 453).

8. Heinrich Toussaint, Verlorene Jahre, (Schicksale einer Kriegsgeneration im Grenzland, 1), Eupen, Grenz-Echo-Verlag, 1987 ; ID., Bittere Erfahrungen, (Schicksale einer Kriegsgeneration im Grenzland, 2), Eupen, Grenz-Echo-Verlag, Eupen, 1987. 
"nouvelle patrie " belge. On y constate le repli dans l'espace religieux et dans des formes de deuil s'abstenant de toute appréciation politique - on honore les morts de toutes les nations. Il soulève également le problème de l'intégration des "survivants ", frères ou fils des soldats allemands, dans l'armée belge ${ }^{9}$. Jusqu'en 1939, l'armée belge réussit plutôt bien cet examen, d'abord en ne réclamant pas pour des raisons psychologiques jusqu'en 1923 le service militaire, puis en garantissant l'instruction du soldat en langue allemande - du moins quand le nombre d'officiers maitrisant la langue le permet. Entre-temps, le conflit entre le camp proallemand (ou " révisionniste " par référence à la soi-disant consultation populaire sur le changement de nationalité en 1920) et le camp probelge s'est radicalisé au sein même du territoire, conduisant à une " barrière de communication ${ }^{10}$. On peut cependant observer que les fils de familles proallemandes notoires effectuent généralement leur service militaire belge sans trop de difficultés; le nombre d'incidents est assez insignifiant. Il n'était pas rare même de voir des germanophones effectuer leur service militaire dans une unité francophone; en effet, il n'existe probablement pas de meilleur moyen d'apprendre une autre langue pour ces jeunes gens dont le niveau d'instruction ne dépasse pas le plus souvent l'école primaire ${ }^{11}$. "Celui qui ne fait pas son service militaire n'est pas un vrai homme ", tels sont les motifs que les jeunes soldats des années 1920 et 1930 avançaient pour expliquer que la question de l'armée ne constitue pas alors pas un champ clos du conflit dominant la vie politique et sociale dans les cantons d'Eupen, de Malmedy et de Saint-Vith.

Après la mobilisation d'août 1939, la situation change. L'État-Major général de l'armée réagit : il retire les réservistes mobilisés de leurs unités pour les verser dans les "Troupes auxiliaires d'armée " (TAA), des troupes d'étapes ne possédant que 25 armes par bataillon. Si certains politiques considèrent cette mesure comme contre-productive et si on reversera finalement une grande partie de ces soldats dans leurs unités d'origine, il n'en reste pas moins que la propagande du printemps 1940 s'est amplement servie de ce signe de méfiance si apparent et de ce sigle permettant des jeux de mots (Tiere Aller Art, ou animaux variés). La propagande allemande justifie par cette "humiliation" les nombreuses désertions - on estime à environ $10 \%$ les soldats des trois cantons qui ont fuit le drapeau belge avant mai 1940 - et le passage de la frontière par une grande partie de ces hommes qui s'engageront dans la fameuse unité spéciale allemande du Bau-Lehr-Bataillon z.b.V. 800 «Brandenburg ». En revanche, la propagande belge considère que ces désertions confirment la légitimité des mesures belges et le bien-fondé d'une méfiance à l'égard de ces soldats.

9. Pour ce qui suit, voir Christoph Brüll, «Entre méfiance et intégration. Les germanophones dans l'armée belge 1920-1955 ", in Cahiers belges d'histoire militaire, n 4, 2006, p. 135-167.

10. Heidi Christmann, Presse und gesellschaftliche Kommunikation in Eupen-Malmedy zwischen den beiden Weltkriegen, thèse de doctorat en communication, Munich, 1974.

11. Christoph Brüll, Die deutschsprachigen Einheiten in der belgischen Armee zwischen den beiden Weltkriegen, Saint-Vith, Éd. zvs, 2004, p. 109. 
Pendant la campagne des Dix-Huit jours, le comportement et le loyalisme des soldats germanophones semblent avoir été plutôt honorables. Certes, il y a eu des défections, mais qui semblent être le résultat de la situation générale défavorable à l'armée belge, plutôt que d'une volonté de passer dans les rangs de la Wehrmacht. À la fin du mois de juin 1940, presque tous les soldats sont de retour dans le territoire d'Eupen-Malmedy, une vingtaine étant morts au combat sous l'uniforme belge. En général, l'attente du retour des soldats qui avaient combattu dans l'armée belge semble avoir été un des rares éléments perturbant l'enthousiasme de la population devant la "libération » du territoire par les Allemands.

\section{L'ANNEXION DU TERRITOIRE AU REICH}

\section{ET L'OCTROI DE LA NATIONALITÉ ALLEMANDE}

L'invasion du 10 mai 1940 ne constituait pas vraiment une surprise pour les Belges. Depuis l'automne 1939, on s'attend à une telle attaque dans les milieux gouvernementaux et militaires belges ${ }^{12}$. Début 1940, quelques familles de probelges éminents et de fonctionnaires dans les trois cantons sont évacuées vers l'intérieur du pays. Parmi ceux qui n'ont pas connu cette faveur, nombreux sont ceux qui deviendront les premières victimes des persécutions nazies - plus d'une fois résultant de dénonciations. Parmi eux, le journaliste Henri Michel, directeur du Grenz-Echo, journal catholique probelge de langue allemande, qui passera presque cinq ans au camp de Sachsenhausen, ainsi que le commissaire de police d'Eupen, Fritz Hennes, et le bourgmestre socialiste de Malmedy, Joseph Werson, tous deux morts dans des camps de concentration ${ }^{13}$.

Dans la recherche historique, il y a aujourd'hui un large consensus pour affirmer que le sentiment prédominant dans la population au moment de l'arrivée des troupes allemandes le 10 mai 1940 fut l'enthousiasme. Le rattachement à Allemagne ne se fit pas attendre et eut lieu le 18 mai 1940 par voie d'un décret du Führer. À ce moment, l'armée belge n'avait pas encore capitulé, ce qui aura pour conséquence la non-reconnaissance juridique de cette annexion dans l'immédiat après-guerre ${ }^{14}$. Le Reich n'en restera pas là. Par un décret du ministre de l'Intérieur, il annexe également, en date du 29 mai 1940, dix communes situées à proximité du territoire d'Eupen-Malmedy mais qui n'avaient jamais fait partie de la Prusse ou de 1982.

12. Cf. Jean Vanwelkenhuyzen, Les Avertissements qui venaient de Berlin, Paris/Gembloux, Duculot,

13. Heinrich Toussaint, "Kollaboration und Widerstand ", in Grenzland seit Menschengedenken, Biblio-Cassette 2, Abhängigkeit, $\mathrm{n}^{\circ} 71$.

14. Führererlass du 18.05.1940 et décret d'exécution du 23.05.1940; Reichsgesetzblatt, 1940/I, p. 777 et 803. Les conséquences historiques et juridiques de cette annexion sont analysées par Carlo Lejeune, Die Säuberung, t. I, Ernüchterung, Befreiung, Ungewissheit (1920-1944), Büllingen LexisVerlag, 2005, p. 63-75 et Jacques Wynants, "Les autorités belges et la situation des cantons de l'Est 1940-1944 ", in Bulletin d'information du Centre liégeois d'histoire et d'archéologie militaires, vol. IX, fasc. IV, mars 2004, p. 15-26. 
l'Allemagne. L'argument utilisé est celui de la langue maternelle allemande d'une partie des habitants de ces communes. Depuis la Première Guerre, l'utilisation de l'allemand dans le domaine public était cependant en net recul, c'est ce qu'on a appelé la "romanisation patriotique ${ }^{15}$. En tout, l'Allemagne nazie gagnait 87000 habitants par ces annexions ${ }^{16}$.

Après une semaine sous administration militaire, c'est l'Administration du Reich qui reprend le territoire d'Eupen-Malmedy. Il est incorporé au Regierungspräsidium d'Aix-la-Chapelle sans que celui-ci ne se voie attribuer plus de fonctionnaires pour cette tâche. Le ministère de l'Intérieur fonctionne comme instance d'approbation pour les différents décrets relatifs au rattachement ${ }^{17}$. Sur le plan politique, le territoire est rattaché au Gau Cologne-Aix-la-Chapelle du NSDAP.

Après le départ et/ou la destitution des bourgmestres belges, ce sont d'abord des membres du parti proallemand, le Heimattreue Front ${ }^{18}$, qui reprennent ces fonctions : à Eupen, l'Ortsgruppenleiter Walter Rexroth, les Kreisleiter Wilhelm Buhrke à Malmedy et Franz Genten à Saint-Vith (ces deux derniers seulement jusqu'en automne 1940). Aux échelons administratifs inférieurs, il n'y a pas de modifications significatives ${ }^{19}$.

Les limites administratives sont adaptées au système du Reich. En lieu et place des trois cantons belges, il n'y a plus que deux Kreise allemands. Les deux Landräte Felix Seulen (Eupen) et Heinz Ehmke (Malmedy) ainsi que le Kreisleiter du NSDAP, Gabriel Saal, sont issus de l'intérieur du Reich, ce qui sera finalement le cas de presque tous les bourgmestresfonctionnaires. Cette politique, qui peut être expliquée par une certaine méfiance mais surtout par des considérations pratiques, crée un sentiment de déception auprès des anciens militants de la cause allemande. Seul, le Kreisleiter d'Eupen, Stefan Gierets, est un ancien du HF, mais pas son successeur Karl Herwanger ${ }^{20}$.

Le Heimattreue Front est déjà rattaché au NSDAP dans le courant du mois de mai 1940. Ce rattachement ne concerne toutefois pas ses membres dont l'affiliation au parti nazi n'est pas automatique. La mission du HF est terminée. La mise au pas et l'auto-intégration de la population progressent rapidement. Schärer estime à 7000 le nombre de membres du NSDAP peu avant la libération de la région par les Alliés en septembre 1944. Des organisations telles la Hitlerjugend et la NS-Frauenschaft comptent à peu près le même nombre de membres. La sa connaît également un grand succès et

15. Cf. Walter Pintens, "Historische Betrachtungen über das deutschsprachige Gebiet und über die rechtliche Stellung der deutschen Sprache in Belgien ", in Fred Stevens et Auweele Van Den, Dirk (éds.), Houd voet bij stuk. Xenia iuris historiae G. Van Dievoet oblata, Katholieke Universiteit Leuven, Leuven, 1990, p. 541-560. L'auteur estime que $13 \%$ de la population de ces « dix communes » avaient l'allemand comme langue maternelle en 1930, taux qui diminuera à 0,72 \% en 1947.

16. Schärer, op. cit., p. 79 sq. et 283.

17. Idem, p. 108 .

18. David Mennicken, Die Heimattreue Front. Eine „nationalsozialistische“ Organisation in Belgien (1936-1940), mémoire de master en histoire inédit, Université catholique de Louvain, 2009-2010.

19. Schärer, op. cit., p. 56 sq. et 146.

20. Idem, p. 113 et 125 . 
les 1240 (certaines sources évoquant même le chiffe de 1 500) membres de la Standarte 174 peuvent accueillir le Stabschef sA Viktor Lutze le 18 mai 1941 pour la prestation de serment. La ss semble avoir beaucoup plus de peine dans le recrutement de membres ${ }^{21}$.

Un décret d'Hitler, daté au 23 mai 1940, prévoit l'introduction de la législation allemande et prussienne à partir du $1^{\text {er }}$ septembre $1940^{22}$. Une nouvelle fois, c'est au ministère de l'Intérieur qu'échoit la mise en œuvre. Une ordonnance du 3 septembre 1940 spécifie des dispositions intermédiaires en droit civil en vue de l'adaptation des principes de droit allemands ${ }^{23}$.

La question la plus compliquée est cependant celle de la nationalité. Elle ne trouve un règlement final que par une ordonnance du ministère de l'Intérieur du 23 septembre 1941. Le décret du 23 mai 1940 avait seulement conféré la nationalité allemande "provisoire " aux habitants ${ }^{24}$. Le nouveau droit de nationalité distingue entre deux groupes dans le territoire d'Eupen-Malmedy et des "dix communes ». La grande majorité des habitants acquiert la nationalité allemande. Environ 20000 habitants des "dix communes " deviennent des Deutsche auf Widerruf (" Allemands sur révocation"), parce qu'ils étaient déjà Belges avant le traité de Versailles. Cette révocation est possible dans un délai de dix ans, qui sera cependant levée peu après. Il est remarquable que la nouvelle réglementation n'enlève pas explicitement la nationalité belge. Ainsi, la population va ignorer complètement qu'en vertu du droit international, elle possède en réalité une «double nationalité » 25 .

\section{L'ENRÔLEMENT DANS LA WEHRMACHT : \\ DE L'ENGAGEMENT VOLONTAIRE À L'OBLIGATION}

L’octroi de la nationalité allemande a une autre conséquence lourde : elle introduit le service militaire dans le territoire d'Eupen-Malmedy. Il s'applique aussi bien aux "nouveaux » Allemands qu'aux "Allemands sur révocation ". Ces derniers peuvent même obtenir définitivement la nationalité allemande s'ils se montrent particulièrement méritants au front ${ }^{26}$. Toutefois, certains jeunes hommes portent déjà l'uniforme de l'armée allemande avant septembre 1941. Outre les déserteurs de l'armée belge qui avaient passé la frontière au printemps 1940, il y a également des volontaires qui s'engagent dans la Wehrmacht entre mai 1940 et l'été 1941. Il ressort

21. Idem, p. 140-143 ; Carlo Lejeune, Die Säuberung, t. II, Hysterie, Wiedereingliederung, Assimilierung (1945-1952), Büllingen Lexis-Verlag, 2007, p. 181-189.

22. Schärer, op. cit., p. 133 sq.

23. Reichsgesetzblatt, 1940/I, p. 1222 sq. et l'arrêté d'exécution du 7.8.1941, Reichsgesetzblatt, 1941 I, p. $376 s q$.

24. Reichsgesetzblatt, $1940 / \mathrm{I}$, p. 803.

25. Reichsgesetzblatt, 1942 I, p. 584. Cf. Schärer, op. cit., p. 143-158.

26. Idem, p. 161. 
de rapports belges d'après-guerre que le nombre de volontaires s'élevait à environ 700. Cependant, nous ne savons pas si ce chiffre concerne la seule période précédant l'octroi de la nationalité allemande ou s'il comporte également les volontaires des années de guerre, c'est-à-dire des jeunes qui veulent s'engager avant même l'appel de leur classe de milice ${ }^{27}$.

Les grades conférés par l'armée belge à ces soldats sont seulement confirmés par les Allemands après six mois de service. Il s'agit donc d'une sorte de probation qui s'étend également aux volontaires. Les anciens officiers de l'armée belge sont exclus de l'enrôlement dans la Wehrmacht à la notable exception près de quelques volontaires ${ }^{28}$.

La procédure établie par les Allemands se déroule en quatre étapes :

1) l'enregistrement ;

2) le test d'aptitude ;

3) six mois de service au Reichsarbeitsdienst (RAD) qui ne s'appliquent pas aux hommes qui ont déjà combattu dans l'armée impériale entre 1914 et 1918 ;

4) l'appel sous les drapeaux.

Les autorités allemandes n'attendent d'ailleurs pas la décision sur la nationalité des habitants pour mettre en œuvre cette procédure. Ainsi, l'enregistrement des classes de milice commence en octobre 1940, tandis que les tests d'aptitude prennent cours en décembre 1940. Suivant le plan de mobilisation du Reich, c'est la classe 1922 qui est alors la première concernée, de même que les anciens officiers de l'armée impériale. Au cours de l'année 1941, malgré la création d'un Wehrmeldeamt, le rythme des enregistrements est considérablement ralenti en attendant le règlement de la question de la nationalitée ${ }^{29}$.

Une fois les habitants devenus "Allemands ", la reprise des enregistrements et des tests d'aptitude suit rapidement. À partir de novembre 1941, les jeunes de la classe 1922 étant déjà enrôlés, les hommes des classes 19001921 sont enregistrés et ceux des années 1914 à 1921 testés. Les tests d'aptitude de janvier à mars 1942 concernent alors les classes 1900 à 1913 ainsi que 1923 et 1924. À partir de ce moment, l'adaptation à la mobilisation dans le Reich est achevée ${ }^{30}$.

Afin de ne pas trop peser sur le moral de la population, les enrôlements ne sont pas affichés sur la voie publique - il ne semble pas y avoir eu des listes aux valves des mairies, mais les hommes concernés sont

27. Idem, p. 162 ; Quadflieg, op. cit., p. 68,143 sq. Un document trouvé par l'auteur aux archives de la ville d'Eupen et réalisé dans le courant de l'épuration civique d'après-guerre comporte 96 noms de volontaires pour les années 1940 à 1944. Si la proportion de volontaires ne diffère pas trop du nombre d'habitants, le chiffre avancé de 700 volontaires concernerait alors probablement la période 1940 1944 et non la seule période entre mai 1940 et septembre 1941. Archives de l'État à Eupen, Eupen : Neuzeit, Nr. 1415 (y 11) Dossier : Ausschluss vom Militärdienst (1945-1949), Liste : «Des volontaires à l'armée allemande ». Cela correspond également aux données relevées par Quadflieg dans un échantillon de 263 soldats originaires d'Eupen-Malmedy.

28. Schärer, op. cit., p. 161-162.

29. Quadflieg, op. cit., p. 69-71.

30. Idem, p. 73-74. 
informés directement par les autorités allemandes. On ne peut douter que l'enrôlement dans la Wehrmacht était soumis à un jeu d'influences diverses qui se manifestent dans des différences du taux d'enrôlement qui ne peuvent être expliquées par le seul facteur démographique. Ainsi, dans deux communes du Kreis d'Eupen, Lontzen (2 085 hab. en 1941) et Raeren (3 271 hab.), le taux des enrôlés était respectivement de 6,33\%, et de $16,01 \%$. La même observation peut être faite pour deux communes du Kreis de Malmedy : à Amblève (Amel ; 1769 hab.) et à Saint-Vith (2 703 hab.), où le taux est de 20,91\% et de 9,98\%. Dans ces communes paysannes, l'enrôlement ne signifie pas seulement un danger personnel pour l'appelé, mais souvent d'abord la perte d'une main-d'œuvre dans les fermes de la région. Ces contraintes socio-économiques expliquent aussi pourquoi la pression directe de dirigeants nazis et un climat de dénonciations peuvent régner sur de nombreuses communes ${ }^{31}$. Au total, le taux d'enrôlement s'élève à 12,87 \% pour le Kreis d'Eupen, à 13,64 \% pour les communes wallonnes du Kreis de Malmedy et à 15,47 pour les communes germanophones de ce Kreis $^{32}$.

Initialement, les autorités allemandes avaient également prévu d'enrôler les hommes originaires d'Eupen-Malmedy qui vivaient en Belgique occupée. Des affiches du 14 avril 1942 annoncent l'enrôlement de tous les Allemands nés entre 1908 et 1924 vivant en Belgique, en spécifiant que cette mesure concerne également les habitants des territoires devenus allemands en septembre 1941. On peut imaginer la consternation chez ces derniers dont on peut aisément soupçonner les sentiments antiallemands. Sur intervention du ministère belge de la Justice, l'administration militaire allemande renonce finalement à cette mesure en acceptant l'argument de la nationalité belge de ces personnes (sans s'interroger sur les conséquences en droit international de cette décision). Le Militärbefehlshaber officialisera la renonciation (provisoire) dans une lettre au secrétaire général du ministère de la Justice datée du 30 mai $1944^{33}$.

Le nombre total des enrôlés du territoire d'Eupen-Malmedy est finalement de 8 700. Il y a cependant aussi des réfractaires qui fuient le service dans la Wehrmacht - généralement en passant la frontière avec la Belgique occupée où ils doivent vivre dans la clandestinité pendant les années de guerre. Le nombre de réfractaires est estimé à 430 : 180 pour le Kreis d'Eupen, 230 pour le Kreis de Malmedy. Il semble que le taux de réfractaires ait été légèrement plus élevé dans les communes wallonnes autour de Malmedy ${ }^{34}$. Leur assurance-vie reposait alors sur le silence de leurs proches ${ }^{35}$.

31. Carlo Lejeune et Klauser Klaus-Dieter, Die Säuberung, t. III, Verdrängte Erinnerungen - 340 Zeitzeugen berichten, Büllingen Lexis-Verlag, 2008, p. 133.

32. Lejeune, Säuberung, t. II, p. 179-180.

33. Lejeune, Säuberung, t. I, p. 79.

34. Idem, t. II, p. 180. Ces estimations sont établies sur la base des chiffres donnés par les différentes communes, à l'exception des deux Kreisstädte pour lesquelles on ne possède pas de chiffres précis.

35. Lejeune et Klauser, Säuberung, t. III, p. 139-147 reprend des témoignages sur les réfractaires et le silence qui entourait leur fuite. 
Le taux de réfractaires est par ailleurs un des éléments qui illustrent au mieux la grande différence entre le territoire d'Eupen-Malmedy et les "dix communes". En effet, le recrutement de soldats pour la Wehrmacht dans ces dernières s'avère être particulièrement délicat et loin de connaitre le même succès. Les organisations de Résistance avancent dans des rapports d'après-guerre le chiffre de 624 réfractaires pour les dix communes (et celle de La Calamine, l'ancien Moresnet-Neutre), voire de 671 réfractaires sur 675 conscrits. Un rapport allemand évoque en juillet 1943 la fuite vers la Belgique de 282 des 585 conscrits des classes 1922 à 1925, soit $48 \%{ }^{36}$. À défaut de la découverte de chiffres plus précis, on peut penser que les données allemandes sont plus proches de la réalité. En effet, même si les activités de la Résistance dans les « dix communes » sont nettement plus nombreuses et denses qu'à Eupen-Malmedy, un taux de presque $100 \%$ de réfractaires paraît très improbable et relève plutôt de la mythologie belge de l'après-guerre ${ }^{37}$.

Les autorités allemandes voient très vite la nécessité de différencier les modalités de recrutement. Elles décident de n'enrôler que les classes à partir de l'année 1922, les classes plus âgées sont jugées trop " probelges » et, dès lors, peu utilisables comme soldats dans l'armée allemande. La méfiance généralisée à l'égard des "Allemands sur révocation " se traduit également dans le fait que les tests d'aptitude n'auront lieu qu'à partir de juin 1943, à un moment, où presque la moitié des conscrits potentiels ont déjà gagné la Belgique occupée. De plus, ceux dont le comportement au RAD n'est pas exempt de tout reproche, ne sont pas enrôlés dans la troupe, mais versés dans des organismes tels que l'Organisation Todt ou le NSKK $^{38}$.

À travers le recrutement, on peut constater que le service dans la Wehrmacht est accepté à Eupen-Malmedy comme une conséquence lourde, mais logique de l'annexion par l'Allemagne et de l'octroi de la nationalité allemande. Ce constat ne vaut cependant pas pour les dix communes également rattachées qui avaient toujours été belges et où la résistance à cet enrôlement est extrêmement forte (à certains égards, elle peut être comparée à celle des habitants du Grand-Duché de Luxembourg ${ }^{39}$ ).

\section{AU FRONT}

Nous savons que l'immense majorité des soldats d'Eupen-Malmedy ont fait leur instruction dans des casernes à l'intérieur du Reich avant de gagner le front de l'Est. Cette destination ne peut surprendre. En effet, c'est en Russie que l'armée allemande a le plus gros besoin d'hommes au moment de l'enrôlement. La proportion de ceux qui sont stationnés à

36. Tous les chiffres sont cités par Schärer, op. cit., p. 165.

37. Cf. les remarques chez Quadflieg, op. cit., p. 84-85.

38. Schärer, op. cit., p. 163-164; Quadflieg, op. cit., p. 76-79.

39. Idem, p. 157-159. 
l'Ouest (en Normandie par exemple) est très petite ${ }^{40}$. Nous ne disposons que de très peu d'informations sur le comportement de ces soldats sur le front de l'Est. Les témoignages connus relatent la participation de certains à la bataille de Stalingrad ou au siège de Leningrad, mais le caractère " forcé » de leur engagement et la tendance à la « victimisation » de l'aprèsguerre ont empêché une approche plus sereine du vécu de ces soldats. Leurs récits et, le plus souvent, leurs lettres de campagne contiennent presque exclusivement des descriptions de ce qu'on pourrait appeler la routine de la guerre ${ }^{41}$. Il n'en reste pas moins que ces soldats ont participé à une des entreprises militaires les plus meurtrières de l'histoire, la guerre d'extermination à l'Est dont les crimes de guerre avec participation des soldats de la Wehrmacht ont été analysés par la recherche la plus récente ${ }^{42}$. La question de l'implication personnelle de soldats d'Eupen-Malmedy dans des crimes de guerre doit donc être posée. Quelques rares témoignages font référence à des fusillades en masse de civils en Russie ${ }^{43}$, mais une étude systématique sur base de toutes les sources disponibles reste un desideratum.

Dans ses travaux, Quadflieg analyse un échantillon de 263 soldats originaires de la région, ce qui nous permet de dégager certaines tendances générales. Ainsi, il démontre que $20,7 \%$ des affiliations de ces soldats à une organisation nazie datent d'avant le 10 mai 1940, tandis que 79,3\% de ces affiliations sont effectuées après l'annexion de 1940. Un effet " suiviste " est donc incontestable, toutefois un endoctrinement nazi (le plus souvent au sein des jeunesses hitlériennes ou du DAF) pour un soldat sur cinq (voire 1 sur 4) peut être constaté. Il n'y a que deux cas où une attitude de refus à l'égard de l'autorité allemande avant l'enrôlement est documentée ${ }^{44}$. Sur le plan strictement militaire, les soldats de la région servent essentiellement dans l'armée de terre (87,5\%). Environ $10 \%$ des enrôlés appartiennent à la Luftwaffe. Le taux des engagés dans la Waffen-SS ne s'élève qu'à 1,5\% . Ceux qui se retrouvent dans des troupes sur le front de l'Est passent 38,5\% de leur temps de service dans des unités de combat, 34,8\% dans des unités de soutien et $26,6 \%$ dans l'armée de réserve ${ }^{45}$. Nous possédons des informations sur leur sort pour environ 68,6 \% des cas analysés dans l'échantillon. Le taux des morts au combat est de $21,5 \%$, celui des prisonniers de guerre s'élève à 24,4\%,11,7\% sont libérés du service avant la fin de la guerre, 9,5 \% sont portés disparus et 1,5\% ont déserté la Wehrmacht ${ }^{46}$.

Il est par ailleurs intéressant de noter que, selon les chiffres de Quadflieg, les décorations et promotions pour des soldats d'Eupen-Malmedy

40. Schärer, p. 162.

41. Exemples chez Toussaint Bitte Erfahrungen et Verlorene Jahre. Une étude systématique des lettres de campagne - champ de recherche de plus en plus fréquenté en Allemagne - manque.

42. Nous ne citons que la synthèse du grand projet « Wehrmacht im Vernichtungskrieg » de l'Institut für Zeitgeschichte de Munich : Christian Hartmann, Johannes Hürter, Peter Lieb et Dieter Pohl, Der deutsche Krieg im Osten 1941-1944. Facetten einer Grenzüberschreitung, Oldenbourg Munich, 2009.

43. Lejeune et Klauser, Säuberung, t. III, p. 135 et 139.

44. Quadflieg, op. cit., p. 132-133.

45. Idem, p. 148-149.

46. Idem, p. 150. 
correspondent largement à la moyenne générale de la Wehrmacht et sont nettement plus élevées que pour les soldats luxembourgeois qui constituent le groupe de comparaison de l'historien d'Aix-la-Chapelle ${ }^{47}$.

Les conclusions de Quadflieg sont claires: les soldats d'EupenMalmedy se sont comportés dans la Wehrmacht comme des soldats originaires du Reich. Leur attitude révèle une conformité incontestable ${ }^{48}$. Ce qui fera d'eux un groupe particulier est leur sort d'après-guerre.

\section{LE DIFFICILE RETOUR}

Cinq mille cinq cents : c'est le nombre d'hommes dont on ne possède aucune information sur le sort en date du 8 mai $1945^{49}$. Il faut attendre le $1^{\text {er }}$ août 1945 pour que les autorités belges disposent d'une liste de ceux qui, dorénavant, s'appelleront les " enrôlés de force ». Dans le courant du mois d'août 1945, on estime pour la première fois que 2500 jeunes hommes sont encore prisonniers de guerre dans des camps soviétiques. Et alors que les quelques centaines de soldats prisonniers dans les camps des Alliés occidentaux sont libérés dans le courant de l'été 1945, il faudra attendre encore quelques années avant que les derniers prisonniers ne puissent regagner leur région. Ainsi, en mars 1946, 2724 prisonniers sont revenus, tandis que 2784 se trouvent toujours dans des camps. Un accord de rapatriement belgo-russe ne contribue que très peu à une accélération de la procédure $^{50}$. A la date du 31 décembre 1947, le nombre de rentrés s'élève à 5 417, celui des prisonniers à 106 et celui des disparus à 1 327. Les autorités ont délivré 1298 attestations de décès officielles.

En mai 1945, les autorités belges reconnaissent que la contrainte est la principale raison de l'enrôlement. Toutefois, de nombreux anciens soldats de la Wehrmacht qui reviennent dans la région dans le courant de l'année 1945 se retrouvent pour quelques jours ou quelques semaines à nouveau prisonniers, cette fois-ci dans la prison de Verviers, ville voisine d'Eupen. Huit cent cinquante et un hommes doivent comparaître devant un tribunal militaire (conseil de guerre avec juges militaires assistés d'un magistrat civil) belge en 1946. Un soupçon généralisé semble alors peser sur ces hommes.

Ce n'est là qu'un signe visible du difficile retour de ces soldats dans leur région, dans une société où l'épuration civique ne semble tolérer qu'une version «belge " du passé le plus récent ${ }^{51}$, où dans de nombreux foyers manquera maintenant l'époux, le père, le frère ou le fils. L'histoire sociale et culturelle de cette perte et de ces familles sans hommes reste encore à écrire.

47. Idem, p. 151-154.

48. Idem, p. 158.

49. Pour ce qui suit Lejeune, Säuberung, t. II, p. 204-207.

50. Idem, t. I, p. 82-84.

51. Freddy Cremer, "Als man den aufrechten Gang wieder lernen musste. Von den "Incivique" zu den "Modellbelgiern" ", in: Cremer Fickers et Lejeune, op. cit., p. 99-116. 
L'État belge aura longtemps du mal à aborder la problématique ${ }^{52}$. Dans les négociations bilatérales avec la jeune RFA au cours des années 1950, le sujet est singulièrement absent et, tandis que la France pour les AlsaciensLorrains et le Luxembourg le font respectivement en 1954 et 1967, la Belgique ne créera qu'en 1974 le statut d'"incorporé de force ". Cinq mille anciens soldats et réfractaires au service dans la Wehrmacht reçoivent finalement un statut juridique. Le dédommagement financier ne sera décidé qu'en 1989, alors que la RFA avait déjà mis les moyens financiers à la disposition de l'État belge en 1962.

À la veille de la disparition des derniers concernés et des derniers témoins, nous devons constater que de larges pages de l'histoire des « enrôlés de force " et de leur sort d'après-guerre restent toujours à écrire. Pour la jeune Communauté germanophone de Belgique, seule une approche critique et sereine de ce passé douloureux permettrait de sortir de la logique de "victimisation " qui a trop longtemps dominé le discours politique et social dans la région.

Christoph BrüLL

Université de Liège

52. Nicolas Dewald, L'Indemnisation des enrôlés de force dans l'armée allemande, mémoire de licence en histoire inédit, Université de Liège, 2004-2005. 